\title{
Characteristics of Coherence and Information for the Davydov Soliton Field
}

\author{
Bi Qiao" ${ }^{1}$ Kongzhi Song ${ }^{2}$, Harry E. Ruda ${ }^{3}$ \\ ${ }^{1}$ Physics Department of Wuhan University of Technology, Wuhan, China \\ ${ }^{2}$ Institute of Space Medico-Engineering, Beijing, China \\ ${ }^{3}$ The Center of Advanced Nanotechnology, University of Toronto, Toronto, Canada \\ Email: biqiao@gmail.com
}

Received September 17, 2012; revised October 29, 2012; accepted November 8, 2012

\begin{abstract}
In this work, a generalized Davydov soliton field (DSF) has been investigated. The characteristics of correlation and information for the filed are emphasized and studied. It is a sort of correlation among so called generalized solitons so that the field may appear to some macroscopic quantum properties such as the superconductivity at room temperature. Secondly, the character of information for the field can be described by a nonlinear Liouville equation in the quantum information representation. This provides a basis to express information quality of DSF, which means that DSF can influence or drive object by using (soliton type of) quantum information density. All these two aspects provide a foundation which possibly explains many potential biological functions of human body such as some complex bio-electromagnetic phenomena and their interactions with objects.
\end{abstract}

Keywords: Soliton; Nonlinear Interaction; Biological Field; Quantum Information; Coherence

\section{Introduction}

For many years, the bio-photonics experiments have discovered that the complex human body likes a set of many molecules and atoms to have particle type of discrete characteristic frequency as a stably entire body. This symbolizes human body to have distinctive quantum excitation field. Not only that, the human body in various function status experiments often be measured with visible color light and a variety of electromagnetic waves as well as acoustic wave phenomena [1-7].

Some scholars from the physical point of view to explore this kind of phenomena, and think the characteristic frequency existence due to nonlinear interaction within the system units continuously exchange energy between body and environment, which results in the coherent excitation of organisms with long-range correlations. This means the coherence can be achieved by nonlinear interaction in organism internal subunits. Thereby they put forward entire concepts about human quantization of dissipative structure in self-organization [8-14].

In fact in early 1973 Davydov proposed protein molecules excited "solitary" model of the energy transport. According to this theory, the protein molecule three spiral micro-vibration and lattice distortion of amide-I exciton interactions produce collective excitations as formed soliton, along the helix propagation, so that ATP molecules hydrolyzed to produce energy from one place to another place. It is found in the experiment $(3-7) \times 10^{10}$ $\mathrm{Hz}$ field frequency that soliton resonance light decompose into excitons and local deformation, corresponding to a new band in $1650 \mathrm{~cm}^{-1}$, with amide-I exciton infrared absorption spectra observed on the $1666 \mathrm{~cm}^{-1}$ line. This proves that there is a red shift of $16 \mathrm{~cm}^{-1}$ corresponding to the formation of just soliton bound energy. This shows the new metabolism possibilities in the presence of excitons and phonon interaction as well collective excitation of coherence. However, Davydov soliton in the presence of short duration is serious obstacle to explain it as a basic information transmission unit. After that, Pang Xiaofeng improved and developed Davydov soliton model, and established a frame of biological soliton transmission theory based on his nonlinear quantum theory $[15,16]$.

The above phenomena and explorations demonstrate that the human body is a quantum field system. This quantum field has complex nonlinear elicitors, biological solitons, and its main interaction may be related to bio-electromagnetic field, which is manifested by the presence of sensitive absorption and emission characteristic frequency and complex spectrum. This is the starting point for further research.

\section{Physical Model}

Let us start from the Davydov soliton perspective and extend the Pang Xiaofeng introduced concepts about the 
domain and the human body biological field to DSF. We can take DSF as a dissipative structure of non-equilibrium thermodynamic system. This DSF system consists of generalized solitons or generalized nonlinear excitations, which represents a local oscillation with characteristics of quasi particles and can transmit from a quantum energy level to other quantum energy level. We extend the theoretical model of the domain, and believe that a domain is a generalized nonlinear excitation or generalized soliton, the most important interaction is with the electromagnetic field, and in addition the generalized soliton is through the exchange of virtual bosons (e.g. virtual phonon or virtual photon) to form the non-local correlation. It is this correlation role that can cause entire cooperation of DSF, thus illustrate a macroscopic quantum effect of DSF under the condition of the correlation strength and correlation distance increase.

Consider a strongly correlated situation, then between generalized solitons (may also include a finite object interacted with DSF) there exist exchange of virtual phonon or virtual photon. This forms a harmoniously entire biological field correlation or orderly self-organization whose wave function is quantized as an operator $\phi$. Although the human body system is considered as an open dissipative structure of non-equilibrium thermodynamic system, but if adding the correlation part caused by interaction with the system, then the system can be seen as a total equilibrium system. Thus, according to the second law of thermodynamics, the free energy density tends to minimum.

Local generalized soliton density is square modulus of field operator $|\phi|^{2}$, where $\phi$ can be defined as an order parameter, where $\phi=0$ as disordered situation, $\phi \neq 0$ as orderly condition. When DSF forms disorderly to orderly transition, in near the transformation point $\phi$ should be small, thus the free energy density $F$ can be expanded by $|\phi|^{2}$, i.e.

$$
F=F_{0}+\alpha_{n}\left|\phi_{n}\right|^{2}+\beta_{n}\left|\phi_{n}\right|^{4}+\cdots
$$

Now let one consider two adjacent domains to form correlation by the exchange of virtual bosons (e.g. virtual phonon or virtual photons). We define first order of correlation as $\left(\gamma_{n} \phi_{n}^{+} \phi_{n+1}+\gamma_{n} \phi_{n-1}^{+} \phi_{n}\right)$, and second order of correlation as

$$
\left(\eta_{n} \phi_{n}^{+} \phi_{n+1} \phi_{n^{\prime}}^{+} \phi_{n^{\prime}+1}+\eta_{n} \phi_{n-q}^{+} \phi_{n} \phi_{n^{\prime}-q}^{+} \phi_{n^{\prime}}\right) .
$$

A higher order of correlation also is possible. It is these generalized solitons with various order of correlations produce a highly delocalized coherent field in the human body, which enable the body to become overall highly ordered field. Here $\alpha, \beta, \gamma . \eta$ are the expansion coefficients of free energy density $F$, where $\gamma, \eta$ are relevant with the strength of the correlation; if they are far greater than 1 , then corresponding field is strongly correlated.

Concerning in present calculation easily to bear simplicity and dropping more than 6 higher order terms, the free energy density $F$ is expressed as:

$$
\begin{aligned}
F= & F_{0}+\sum_{n=1}^{N} \alpha_{n}\left|\phi_{n}\right|^{2}+\sum_{n=1}^{N} \beta_{n}\left|\phi_{n}\right|^{4}+\frac{1}{2 m^{*}}|i \nabla \phi(r)|^{2} \\
& +\sum_{n=1}^{N}\left(\gamma_{n} \phi_{n}^{+} \phi_{n+1}+\gamma_{n} \phi_{n-1}^{+} \phi_{n}\right) \\
& +\sum_{n, n^{\prime}=1}^{N}\left(\eta_{n} \phi_{n}^{+} \phi_{n+1} \phi_{n^{\prime}}^{+} \phi_{n^{\prime}+1}+\eta_{n} \phi_{n-q}^{+} \phi_{n} \phi_{n^{\prime}-q}^{+} \phi_{n^{\prime}}\right)+\cdots
\end{aligned}
$$

where $F_{0}$ represents the disorder of the free energy $\phi_{n}=0, N$ is localized particle number. Also consider the generalized coordinates $\phi_{n}$ and the generalized momentum $\phi_{n}$, the Lagrange density is

$$
L=\frac{1}{2} m^{*} \sum_{n-1}^{N} \phi_{n}^{+} \dot{\phi}_{n}+\frac{1}{2} m^{*} \sum_{n-1}^{N} \phi_{n} \dot{\phi}_{n}^{+}-F
$$

where the former two are the kinetic energy of the system, and $F$ is equivalent to the potential energy.

In the continuous approximation, taking minimum of equilibrium free energy functional as $\frac{\delta F}{\delta \varphi}=0$, the Euler equation is given by

$$
\begin{aligned}
& i \mu \frac{\partial \phi(t)}{\partial t}=(\alpha-2 \gamma) \phi(t)-\alpha \gamma \nabla^{2} \phi(t) \\
& +2 \beta|\phi(t)|^{2} \phi(t)+4 \eta|\phi(t)|^{4} \phi(t)
\end{aligned}
$$

where $i \mu=m^{*}$. This is a non-linear Schrodinger equation, which can be viewed as an extension of four orders for the soliton equation proposed by Pang Xiaofeng at ATP hydrolysis in helix proteins producing collective excitations [15-17]. Hence, this equation can be defined as a basic equation describing DSF in highly correlated states. In considering the human field high order coherent conditions, higher order of extension of this equation is also possible, in that situation the solution is usually associated with multiple well potentials.

In fact for the second order of approximation, the soliton type of solution is given by [17]

$$
\begin{aligned}
& \phi(r, t)=\left(\frac{\alpha}{\beta}\right)^{\frac{1}{2}} \operatorname{sech}\left\{\sqrt{\lambda}\left[\frac{x}{\alpha \sqrt{\lambda}}-v_{g}\left(t-t_{0}\right) / \sqrt{m^{*}}\right]\right\} \\
& \cdot \exp i\left\{\frac{v_{g}}{2 \alpha \sqrt{\lambda}}+\left[\lambda-\frac{v_{g}^{2}}{4}-(\alpha-2 \gamma) \frac{t}{\sqrt{m^{*}}}\right]\right\}
\end{aligned}
$$

where the envelop wave is

$$
\operatorname{sech}\left\{\sqrt{\lambda}\left[\frac{x}{\alpha \sqrt{\lambda}}-v_{g}\left(t-t_{0}\right) / \sqrt{m^{*}}\right]\right\},
$$


the velocity of wave is $v_{g}$, and the carrier is as a harmonic wave

$$
\exp i\left\{\frac{v_{g}}{2 \alpha \sqrt{\lambda}}+\left[\lambda-\frac{v_{g}{ }^{2}}{4}-(\alpha-2 \gamma) \frac{t}{\sqrt{m^{*}}}\right]\right\} .
$$

Thus the introduction $\phi(r, t)$ as order parameter $=$ biological field. The order parameter (field operator) $\phi(r, t)$ composes of DSF which describes cooperative behaviors of many particles as an entirety; therefore it is called as macroscopic wave function or order parameter based on the Landau second type of phase transition theory [18]. This highly ordered biological field can be further integrated with only one field function expression $\phi$ and keep step with consistent with overall vibration to show macroscopic quantum effect that can be seen as an origin of many human biological function or potential special features of nonlinear quantum mechanics.

In short, biological DSF systems rely on the non-linear interactions among internal sub-units and the energy exchange process of self-organization with environment to maintain their order and perfect ceaselessly, and form its own characteristic frequency. These presences of multiple biological elicitor and soliton may be key mechanism to support a variety of special features of the human body functions. These biological elicitor and soliton in the body cycle tracks are probably so called acupuncture channels.

\section{Superconductivity in Room Temperature}

One interesting notice here is that the above second-order approximation equation corresponding to the potential field can be expressed as:

$$
U(r, t)=(\alpha-2 \gamma)|\phi(r, t)|^{2}+2 \beta|\phi(r, t)|^{4}
$$

It is a double potential well (because the original equation is of the second-order), there are two minima for

$$
\phi_{0}(r, t)= \pm \sqrt{\frac{-(\alpha-2 \gamma)}{4 \beta}}
$$

Not in the point $\phi_{0}(r, t)=0$. If $\alpha>2 \gamma$, then $\beta<0$, then it gives

$$
\phi_{0}(r, t)=\left\{\begin{array}{l}
\phi_{0}^{1}=\sqrt{\frac{-(\alpha-2 \gamma)}{4 \beta}} \\
\phi_{0}^{2}=-\sqrt{\frac{-(\alpha-2 \gamma)}{4 \beta}}
\end{array}\right.
$$

There is clearly to show these ground state changes have the symmetry broken, which has consistency same as the situation of electro-acoustic superconductor system.

In fact in the electro-acoustic superconductors, when the electrons in the two equivalent ground states, the above two energy minima are just ground states of the super-conductor because the electron and virtual phonon nonlinear interactions prevent electron energy dispersion and thereby form a stable superconducting electronicCooper pair. The corresponding energy represents as $\frac{2 r \alpha \sqrt{\gamma r}}{\beta}$, which is just a binding energy for the electronic bound of the Bose-Cooper pair. So this Cooper pair is not else, but a kind of soliton. Hence the superconducting electrons are also to form a kind of solitons. Pang Xiaofeng has pointed out this in [17].

We want to emphasize here the above similarity between the theoretical model of superconductivity and the correlation model of DSF. Superconducting critical temperature can be obtained by considering the soliton formula, the finite temperature gap equation and the quasiFermi particle distribution [19],

$$
T_{c} \propto 1.14 \omega_{p} \mathrm{e}^{-\frac{1}{\eta g(0)}}
$$

where $g(0)$ is the density of states. The key is the nonlinear coupling coefficient $\gamma$ in the nonlinear interaction $\eta|\phi|^{4}$ of Equation (4). If $\eta$ becomes bigger, superconducting transition temperature increases. It is precisely $\eta$ influencing the correlation strength enhancement, so that the superconducting transition temperature is increased. This demonstrates that the superconductivity for DSF model even at room temperature is possible.

But the high temperature superconducting mechanism and theory has not finally been settled, and the room temperature superconductor effect either experimentally or theoretically is very thorny problem. Moreover, the hypothesis as superconductive electrons form a solitary is also required to confirm in relevant experiments of high temperature superconductor. Also from experimental perspective, human superconducting phenomenon has not been found, and some experiments only discovered that the acupuncture channels resistance is smaller than other direction resistor. Whether or not there exist some special functional states whose resistance closes to zero? This sort of experiment needs to be investigated in future.

Thus, we suggest performing an experiment in which DSF is applied to the appropriate materials to induce room temperature superconductor. If this can be realized, then in a considerable extent it demonstrates that our proposed above generalized soliton field theory is a key mechanism to explain many phenomena of the bio-electromagnetic experiments, at the same time, also gives quit important contribution for the high (room) temperature superconducting mechanism.

\section{Biological Information Field}

The above Equation (4) is for describing DSF in human 
body, whose solution is a kind of generalized biological soliton under certain conditions which can propagate over distance without decaying inside or outside the human body. These solitons are considered to be the basic units of human biological information transmission. This introduces a biological information problem in human body. Further, DSF can also be generalized to a kind of biological information field. We can even consider what information density equation to drive or control DSF and produces some special physiological functions above the threshold of energy information intensity, because in the real world, there may exist effective interaction between human body biological field and environment, such as a variety of spectroscopy, magnetic spectrum radiation treatment as a role of physiotherapy instrument for human body $[20,21]$.

Here character of information for DSF may be described by an information representation of the nonlinear Liouville equation [22-25]. To explain clearly, we firstly define a quantum information density as

$$
I=\rho \ln \rho
$$

Then the information representation of quantum Liuville equation is deduced by following calculations:

$$
\begin{aligned}
& i \frac{\partial}{\partial} \rho^{2}=i\left(\frac{\partial \rho}{\partial t}\right) \rho+i \rho\left(\frac{\partial \rho}{\partial t}\right) \\
& =[H, \rho] \rho+\rho[H, \rho]=\left[H, \rho^{2}\right] \\
& \ldots \\
& i \frac{\partial}{\partial} \rho^{n}=\left[H, \rho^{n-1}\right] \rho+\rho^{n-1}[H, \rho] \\
& =\left[H, \rho^{n}\right] \text { for any integer } n,
\end{aligned}
$$

which illustrates that a quantum information density (QID), $I=\rho \ln \rho$, to meet the Liouville equation by the power series of $\rho$ expanding as

$$
i \frac{\partial I}{\partial t}=L I=[H, I]
$$

Furthermore, for a classical system it can also be proved that the classical Liouville equation can be written by [25].

Therefore, the description of the generalized soliton can be extended into a concept of the generalized quantum information density, since the corresponding nonlinear Schrodinger equation stunned into generalized Liouville equation given by

$$
i \frac{\partial \rho}{\partial t}=L \rho+R[\rho]
$$

where $R[\rho]$ is the nonlinear self-interaction term. Below, we deduce some specific forms of $R[\rho]$ explicitly.

Suppose that a general expression for the distribution function is given by

$$
\rho=\mathrm{e}^{F}
$$

The corresponding non-equilibrium Liouville equation is

$$
\frac{\mathrm{d} \rho}{\mathrm{d} t}=i \frac{\partial \rho}{\partial t}-L \rho=R[\rho]
$$

For example:

$$
i \frac{\partial \rho}{\partial t}=L \rho+\mathrm{e}^{\rho}
$$

According to the above point of view, more broadly, whole world is composed of quantum information density driven by energy, and all kinds of objects only presented various information density distribution. Often this information distribution at different frequency, with various waveform amplitudes, but by the driving from a type of quantum soliton information density field, the target information distribution with different frequency and waveform amplitudes will tend to a synchronous resonance, so that the target information density distribution become the same distribution as the quantum soliton information density field. The both become an entire form to possess possibly macroscopic quantum effect to render many specific potential functions of human body.

For example: by introducing a quantum information

$$
\begin{aligned}
i \frac{\partial \rho \ln \rho}{\partial t} & =i\left(\frac{\partial \rho}{\partial t}\right) \ln \rho+i \rho\left(\frac{\partial \rho}{\rho \partial t}\right)=\{H, \rho\} \ln \rho+\{H, \rho\} \\
& =\frac{\partial H}{\partial p}\left(\frac{\partial \rho}{\partial q}\right) \ln \rho-\frac{\partial H}{\partial q}\left(\frac{\partial \rho}{\partial p}\right) \ln \rho+\frac{\partial H}{\partial p} \frac{\rho \partial \rho}{\rho \partial q}-\frac{\partial H}{\partial q} \frac{\rho \partial \rho}{\rho \partial p} \\
& =\frac{\partial H}{\partial p}\left(\frac{\partial \rho}{\partial q}\right) \ln \rho-\frac{\partial H}{\partial q}\left(\frac{\partial \rho}{\partial p}\right) \ln \rho+\frac{\partial H}{\partial p} \frac{\rho \partial \ln \rho}{\partial q}-\frac{\partial H}{\partial q} \frac{\rho \partial \ln \rho}{\partial p} \\
& =\frac{\partial H}{\partial p}\left[\left(\frac{\partial \rho}{\partial q}\right) \ln \rho+\frac{\rho \partial \ln \rho}{\partial q}\right]-\frac{\partial H}{\partial q}\left[\left(\frac{\partial \rho}{\partial p}\right) \ln \rho+\frac{\rho \partial \ln \rho}{\partial p}\right] \\
& =\frac{\partial H}{\partial p} \frac{\partial \rho \ln \rho}{\partial q}-\frac{\partial H}{\partial q} \frac{\partial \rho \ln \rho}{\partial p}=\{H, \rho \ln \rho\}
\end{aligned}
$$


density potential (PQID), make the human biological information field to drive an object:

$$
\rho \rightarrow \rho_{o b}
$$

Thus, an equation of information density of the biological field and the object is entirely written as:

$$
i \frac{\partial \rho}{\partial t}=L \rho+\frac{1}{2} V(\rho)
$$

where $\rho \propto \rho_{o b}$.

Then the use of Baker-Hausdorff formula and Magnus lemma [26], one gets

$$
\rho \frac{\mathrm{d} \ln \rho}{\mathrm{d} t}=\left\{V(\rho), \frac{\ln \rho}{1-\rho}\right\}
$$

where defining

$$
\left\{x, y^{n}\right\} \equiv[[\cdots[x, y], y] \cdots, y]
$$

Therefore one obtains

$$
\begin{aligned}
& \frac{\mathrm{d}}{\mathrm{d} t} \rho \ln \rho=\frac{\mathrm{d} \rho}{\mathrm{d} t} \ln \rho+\rho \frac{\mathrm{d} \ln \rho}{\mathrm{d} t} \\
& =V(\rho) \ln \rho+\left\{V(\rho), \frac{\ln \rho}{1-\rho}\right\}
\end{aligned}
$$

Introducing the non-equilibrium and irreversible condition, having

$$
\frac{\mathrm{d}}{\mathrm{d} t} \rho \ln \rho=V(\rho) \ln \rho+\left\{V(\rho), \frac{\ln \rho}{1-\rho}\right\} \neq 0
$$

which enables one to have

$$
V(\rho) \ln \rho \neq 0
$$

and

$$
\left\{V(\rho), \frac{\ln \rho}{1-\rho}\right\}=0
$$

This allows one to obtain the type of PQID as

$$
V(\rho)=V\left(\frac{\ln \rho}{1-\rho}\right)
$$

were $V$ is a functional of $\frac{\ln \rho}{1-\rho}$, and a simple form can be

$$
V(\rho)=\frac{\ln \rho}{1-\rho}
$$

this shows $\rho \in[0,1)$, and $V(\rho)$ is analytic, i.e.

$$
\begin{aligned}
& \frac{\ln \rho}{1-\rho}=-1+\frac{1}{2}(\rho-1)-\frac{1}{3}(\rho-1)^{2} \\
& +\cdots-(-1)^{n+2} \frac{1}{n}(\rho-1)^{n-1}-\cdots
\end{aligned}
$$

This $V(\rho)$ can be analytic function for any $\rho \in[0,1)$, and can commute with $\frac{\ln \rho}{1-\rho}$, and satisfies condition (111), which establishes a general nonlinear Liouville equation as

$$
i \frac{\partial \rho}{\partial t}-L \rho=V(\rho)
$$

The above type of Liouville equation may have soliton type of solution. The physical meaning can be considered that this equation consists of a kind of nonlinear Schrödinger equations. For instance, for a pure ensemble system, $\rho=|\varphi\rangle\langle\varphi|$, if constructing $R=\rho^{2}$, then one gets

$$
\begin{aligned}
i \frac{\partial \rho}{\partial t} & =\left[H_{0}, \rho\right]-\rho^{2} \\
& =H_{0} \rho-\rho H_{0}-|\varphi\rangle|\varphi|^{2}\langle\varphi| \\
& =H_{0} \rho-\rho H_{0}-\frac{1}{2}|\varphi\rangle|\varphi|^{2}\left\langle\varphi\left|-\frac{1}{2}\right| \varphi\right\rangle|\varphi|^{2}\langle\varphi|
\end{aligned}
$$

which gives the nonlinear Schrödinger equation described as

$$
\begin{aligned}
& i \frac{\partial|\varphi\rangle}{\partial t}=H_{0}|\varphi\rangle-\frac{1}{2}|\varphi\rangle|\varphi|^{2}, \\
& -i \frac{\partial\langle\varphi|}{\partial t}=\left\langle\left.\varphi\left|H_{0}-\frac{1}{2}\right| \varphi\right|^{2}\langle\varphi|\right.
\end{aligned}
$$

where notice the nonlinear part of the Hamiltonian is not self-adjoint, showing the system may be an open system with the evolution symmetric broken. This shows the above equation with $R(\rho)=\rho^{2}$ is related to the solitons through the nonlinear Schrödinger equations mentioned. However, it is not restricted the solitons since the nonlinear term $R(\rho)$ could be functional of $\rho$, such as $\exp (\rho), \rho \ln \rho, \operatorname{sech}(\rho) \exp (\rho)$, and so on, which may describe more general nonlinear interactions.

As an example of the application, suppose that a human biological field is described by

$$
i \frac{\partial \rho}{\partial t}=\left[H_{0}, \rho\right]-\lambda \mathrm{e}^{\rho}
$$

where $\lambda \ll 1$ is quite week introduced by the field subjects to the attack such as sick, disturbing, etc. Then one can use the nonlinear series of pulses (e.g. from medical device) such as $\mathrm{e}^{\omega_{1} t} \rho, \mathrm{e}^{\omega_{2} t} \rho, \mathrm{e}^{\omega_{3} t} \rho, \cdots$, to allows

$$
\kappa \mathrm{e}^{\rho}=\sum_{n} \mathrm{e}^{\omega_{n} t} \rho^{n}
$$

so that

$$
\begin{aligned}
i \frac{\partial \rho}{\partial t} & =\left[H_{0}, \rho\right]-\lambda \mathrm{e}^{\rho}-\sum_{n} \mathrm{e}^{\omega_{n} t} \rho^{n} \\
& =\left[H_{0}, \rho\right]-(\lambda+\kappa) \mathrm{e}^{\rho}
\end{aligned}
$$


where $\lambda+\kappa \gg 1$ is magnified, which enables the system to return to health state by agitating. A concrete driving pulse function can be considered as

$$
\left|f_{m}\right\rangle=A_{m} \mathrm{e}^{i\left(\omega_{m} t+\varphi_{m}\right)}|m\rangle
$$

and a corresponding density operator is

$$
\begin{aligned}
\left|f_{m}(t)\right\rangle\left\langle f_{n}(t)\right| & =A_{m n} \mathrm{e}^{i\left(\omega_{m n} t+\varphi_{m n}\right)} \alpha_{m n}|m\rangle\langle n| \\
& =A_{m n} \mathrm{e}^{i\left(\omega_{m n} t+\varphi_{m n}\right)} \alpha_{m n} \rho
\end{aligned}
$$

where $\alpha_{m n}$ is a transition from $m^{\text {th }}$ state to $n^{\text {th }}$ state, $\phi_{m}$ is an initial phase for $m$ state, $\omega_{m n}$ is a frequency which is related to $m$ and $n$ states. Therefore a series of multiplied pulses can be constructed by

$$
\mathrm{e}^{\omega_{1} t+\varphi}, \mathrm{e}^{2\left(\omega_{2} t+\varphi\right)}, \mathrm{e}^{3\left(\omega_{2} t+\varphi\right)}, \cdots
$$

which allows a driving PQID $f(\rho)$ from an external source to be expressed by

$$
f(\rho)=\kappa \sum_{n} \mathrm{e}^{n\left(\omega_{n} t+\varphi_{n}\right)} \rho^{n}
$$

and is proportional to the original $V(\rho)=\lambda \mathrm{e}^{\rho}$ by adjusting frequency $\omega_{n}$. Here $f(\rho)$ is an analytic function of $\rho$. For instance, by choosing

$$
\omega_{n}=\frac{1}{n t} \ln \frac{1}{n !}-\frac{\phi}{t},
$$

such as

$$
\begin{aligned}
& \omega_{0}=-\frac{\phi}{t}, \omega_{1}=-\frac{\phi}{t}, \\
& \omega_{2}=\frac{1}{2 t} \ln \frac{1}{2}-\frac{\phi}{t}, \omega_{3}=\frac{1}{3 t} \ln \frac{1}{6}-\frac{\phi}{t}, \\
& \omega_{4}=\frac{1}{4 t} \ln \frac{1}{24}-\frac{\phi}{t}, \omega_{5}=\frac{1}{5 t} \ln \frac{1}{120}-\frac{\phi}{t}, \cdots,
\end{aligned}
$$

so that $\lambda \mathrm{e}^{\rho}$ can be magnified by the driving PQID $f(\rho)$, namely for any $\kappa \gg 1$,

$$
\begin{aligned}
f(\rho) & =\kappa \exp (\rho) \\
& =\kappa\left[1+\rho+\frac{1}{2} \rho^{2}+\frac{1}{6} \rho^{3}+\frac{1}{24} \rho^{4}+o\left(\rho^{5}\right)\right]
\end{aligned}
$$

In brief, by considering the significance of $\rho$ as a minimum information density, generally speaking, the new equation driving object information density $\rho_{o b}$ has a solitary type of solution, which provides certain PQID to drive the quantum information density of object to tend to a kind of resonance synchronous, so that the both become highly coherent as information solitons with a variety of macroscopic quantum effect. Extremely, it is possible that the information wave (envelope) to around the object form an entire soliton, so that the carrier of waves in the solitons have resonance with each others as a sort of strongly non-local correlations. This may make the target atom or molecule separation, aggregation, displacement and finally accompanied by the wave carrier strength reaching a threshold, some macro-quantum effects (such as macro-quantum tunnelling, the room superconductor phenomenon, etc.) can be shown as mentioned in previous description.

\section{Conclusion}

The coherence and information aspects for DSF theory have been investigated. We firstly emphasize the correlation characteristics of the filed. It is this sort of correlation between so called generalized solitons, some macroscopic quantum effects are possible such as the superconducting at room temperature. Secondly, a nonlinear Liouville equation in the quantum information representation has been represented, which provides a basis to describe information character of DSF. This means that DSF can be influenced or driven by object using (soliton type of) PQID. All these two elements provide a foundation possibly to explain many potential functions of human body and relevant interactions with objects.

\section{Acknowledgements}

This work was supported by the grants from Canada by NSERC, MITACS, CIPI, MMO, and CITO.

\section{REFERENCES}

[1] P. N. Prasad, "Introduction to Biophotonics," Wiley-Interscience, New York, 2003. doi:10.1002/0471465380

[2] K. Z. Song, "The Existence and Significance of Parapsychological Function," Journal of International Society of Life Information Science (ISLIS), Vol. 17, No. 1, 1999, pp. 198-214.

[3] Q. Y. Wu, et al., "Research of Electromagnetic Radiation from Human Body," Chinese Journal of Somatic Science, Vol. 3, No. 4, 1991, pp. 166-167.

[4] B. J. Wu, et al., "The Dynamic Study of Magnetic Signal Frequency-Power Spectrum from Different Points by Superconducting Biomagnetometer," Chinese Journal of Somatic Science, Vol. 4, No. 2, 1994, pp. 81-82.

[5] K. Z. Song, "The Research on Characteristics and Physiological Foundation of Human Body Parapsychological Function," Chinese Journal of Somatic Science, Vol. 5, No. 4, 1995, pp. 147-155.

[6] J. C. Shen, et al., "Examination and Thinking of SoulControlled Energy Gathering by Sun Chulin," Chinese Journal of Somatic Science, Vol. 6, No. 1, 1996, pp. 1015.

[7] J. C. Shen, et al., "To Explore the Secret of Human Body Magnetic Field-The Quantitative Determination of the Human Body Magnetic Field under the Qigong State," Chinese Journal of Somatic Science, Vol. 7, No. 1, 1997, pp. 3-7. 
[8] H. J. Irwin and C. A. Watt, "An Introduction to Parapsychology," 5th Edition, Mcfarland \& Company, Inc., Jefferson, 2007, pp. 124-137.

[9] F. A. Popp, H. L. Kong, et al., "Electromagnetic BioInformation," 2nd Edition, Urban \& Schwarzenberg, Munchen-Wien-Baltimore, 1989.

[10] Y. Xiao and X. Z. Lin, "Soliton Model for Energy Transport in Collagen Molecules," Communications in Theoretical Physics, Vol. 28, No. 2, 1997, pp. 253-256.

[11] Y. Xiao and X. Z. Lin, "Nonlinear Self-Localized Model in a Chain of Two-Level Molecules," Journal of Physics A: Mathematical General, Vol. 29, No. 10, 1996, pp. 26112615. doi:10.1088/0305-4470/29/10/036

[12] H. Frohlich, "Coherent Exitation in Biological Systems," Springer-Verlag, Berlin, 1982.

[13] H. Frohlich, "Biological Coherence and Response to Exteral Stimuli," Springer-Verlag, Berlin, 1988. doi:10.1007/978-3-642-73309-3

[14] F. A. Popp, "Electromagnetic Bio-Information," In: C. W. Smith, Ed., Coherent Electromagnetic Fields and Bio-Communication, Urban \& Schwarzenlerg, Baltimore, 1989, pp. 1-17.

[15] X. F. Pang, "Nonlinear Quantum Mechanics," Publishing House of Electronics Industry, Beijing, 2009.

[16] X. F. Pang and Y.-P. Feng, "Quantum Mechanics in Nonlinear Systems," World Scientific Publishing Co. Pte. Ltd., Singapore City, 2005.
[17] X. F. Pang, "Nonlinear Quantum Mechanics," Chongqing Press, Chongqing, 1993.

[18] L. D. Landau, "Quantum Mechanics," Pergamon Press, Oxford, 1987.

[19] Z. Z. Li, "Solid Theory," Advanced Education Press, 2006.

[20] B. Wilson, V. V. Tuchin and S. Tanev, "Advances in Biophotonics," NATO Press, Ottawa, 2005.

[21] X. Shen and R. van Wijk, "Biophotonics: Optical Science and Engineering for the 21st Century," Springer, New York, 2005.

[22] Q. Bi, X. S. Xing and H. E. Ruda, "Dynamical Equations for Quantum Information and Application in Information Channel," Chinese Physics Letters, Vol. 22, No. 7, 2005, p. 1618. doi:10.1088/0256-307X/22/7/016

[23] Q. Bi and J. Q. Fang, "Network Science and Method of statistical Physics," Beijing University Press, Beijing, 2011.

[24] Q. Bi, H. E. Ruda and Z. D. Zhou, "Dynamical Equations of Quantum Information and Gaussian Channel," Physica A, Vol. 363, No. 2, 2006, p. 198. doi:10.1016/j.physa.2005.08.044

[25] Q. Bi, Fang J. Q. and H. E. Ruda, "Some Charicteristics and Applications for Quantum Information," Journal of Modern Physics, Vol. 3, No. 9, 2012, pp. 1070-1080. doi:10.4236/jmp.2012.39141

[26] B. C. Eu, "Nonequilibrium Statistical Mechanics (Ensemble Method)," Kluwer Academic Publishers, Dordrecht, Boston, London, 1998. 